\title{
Elimination of p19ARF-expressing cells enhances pulmonary function in mice
}

\author{
Michihiro Hashimoto, ${ }^{1}$ Azusa Asai, ${ }^{1}$ Hiroyuki Kawagishi, ${ }^{1}$ Ryuta Mikawa,, ${ }^{1}$ Yuji Iwashita, ${ }^{1}$ \\ Kazuki Kanayama, ${ }^{2}$ Kazushi Sugimoto, ${ }^{3}$ Tadashi Sato, ${ }^{4}$ Mitsuo Maruyama, ${ }^{1}$ \\ and Masataka Sugimoto ${ }^{1}$ \\ 'Department of Mechanism of Aging, National Center for Geriatrics and Gerontology, Obu, Aichi, Japan. ${ }^{2}$ Department of \\ Oncologic Pathology and ${ }^{3}$ Department of Molecular and Laboratory Medicine, Mie University School of Medicine, \\ Tsu, Mie, Japan. ${ }^{4}$ Department of Respiratory Medicine, Juntendo University School of Medicine, Tokyo, Japan.
}

\begin{abstract}
Senescent cells accumulate in many tissues as animals age and are considered to underlie several aging-associated pathologies. The tumor suppressors p19ARF and p16 ${ }^{\text {INK4a }}$, both of which are encoded in the CDKN2A locus, play critical roles in inducing and maintaining permanent cell cycle arrest during cellular senescence. Although the elimination of $\mathrm{p} 16^{\mathrm{INK} 4 \mathrm{a}}$-expressing cells extends the life span of the mouse, it is unclear whether tissue function is restored by the elimination of senescent cells in aged animals and whether and how p19ARF contributes to tissue aging. The aging-associated decline in lung function is characterized by an increase in compliance as well as pathogenic susceptibility to pulmonary diseases. We herein demonstrated that pulmonary function in 12-month-old mice was reversibly restored by the elimination of p19ARF-expressing cells. The ablation of p19ARF-expressing cells using a toxin receptor-mediated cell knockout system ameliorated aging-associated lung hypofunction. Furthermore, the aging-associated gene expression profile was reversed after the elimination of $\mathrm{p} 19^{\mathrm{ARF}}$. Our results indicate that the agingassociated decline in lung function was, at least partly, attributed to $\mathrm{p} 19^{\mathrm{ARF}}$ and was recovered by eliminating p19 ${ }^{\mathrm{ARF}}$-expressing cells.
\end{abstract}

Conflict of interest: The authors have declared that no conflict of interest exists.

Submitted: April 1, 2016 Accepted: June 23, 2016 Published: August 4, 2016

Reference information: JCI Insight. 2016;1(12):e87732. doi:10.1172/jci.insight.87732.

\section{Introduction}

Most mammalian somatic cells have infinite replicative life spans and eventually undergo permanent cell cycle arrest, called cellular senescence (1). Senescence is triggered by sustained and irreparable damage that leads to the activation of tumor suppressor pathways. Two major tumor suppressor pathways, namely, the p19 ${ }^{\mathrm{ARF}}$ (p14 $4^{\mathrm{ARF}}$ in humans)/p53 and $\mathrm{p} 16^{\mathrm{INK} 4 \mathrm{a}} / \mathrm{Rb}$ pathways, play critical roles in the induction and maintenance of cell cycle arrest during cellular senescence $(2,3)$. The inactivation of these pathways bypasses senescence, thereby allowing the propagation of damaged cells, which eventually leads to cancer. Thus, cellular senescence eliminates potential malignant transformation and functions as an essential tumorsuppressive mechanism in mammals.

While there is no doubt that senescence prevents cancer, an increasing amount of evidence suggests that cellular senescence is involved in other biological processes and pathologies. Cellular senescence has been shown to contribute to embryonic development (4, 5), wound healing (6), and tissue regeneration (7). Additionally, it has become more evident that cellular senescence contributes to tissue aging. Senescent cells accumulate in many tissues during aging (8) and are considered to underlie aging-associated pathologies (9). The contribution of senescent cells in aging-associated phenotypes may depend on their non-cellautonomous functions, such as senescence-associated secretory phenotype (SASP), because the population of senescent cells is very small, even in very old human tissue (10).

Senescence is enhanced in BubR1 mutant mice that show accelerated aging phenotypes (11), and the lifelong elimination of $\mathrm{p} 16^{\mathrm{INK} 4 \mathrm{a}}$-expressing senescent cells from these mice was found to partially reverse these phenotypes (11). The ablation of cellular senescence by deleting the INK4a gene also restored some progeria-like phenotypes and extended the life span of Klotho mutant mice (12). Collectively, these findings indicate that cellular senescence is responsible for aging-associated phenotypes but may not account for all phenomena in aged animals. It has not yet been established whether cellular senescence contributes to chronological (naturally occurring) aging phenotypes and, more importantly, if aging-associated pheno- 
types may be reversed by eliminating senescent cells from old animals. van Deursen and colleagues recently reported that the clearance of senescent cells in old animals extended the life span of the mouse using an INK-ATTAC transgenic model (13). They concluded that age-dependent changes, at least in the kidney, heart, and adipose tissue, are caused by $16^{\text {INK4a }}$-expressing senescent cells, which strongly influence the life span of this animal.

In the present study, we established a transgenic model from which it was possible to eliminate p19 ${ }^{\mathrm{ARF}}$-expressing cells using a toxin-mediated cell knockout system $(14,15)$. Similar to INK4a, the expression of $A R F$ has been shown to increase during aging in the mouse (8). Using the transgenic model, we successfully eliminated $A R F$-expressing cells from the lung tissue of 12-month-old animals. The ablation of $A R F$ expression abolished the expression of other senescent markers, including INK4a and $p 21$, suggesting that the expression of $A R F$ reflects the accumulation of senescent cells in tissues. The elimination of $\mathrm{p} 19^{\mathrm{ARF}}$-expressing cells in lung tissue ameliorates the aging-associated loss of tissue elasticity. Moreover, the expression of a large number of aging-associated genes was reversed after the removal of $\mathrm{p} 19^{\mathrm{ARF}}$-expressing cells. Taken together, these findings highlight the role of $\mathrm{p} 19^{\mathrm{ARF}}$ in lung tissue aging and indicate that the aging phenotype in lung tissue may be reversed by eliminating $\mathrm{p} 19^{\mathrm{ARF}}$. expressing cells from tissue.

\section{Results}

In order to investigate the role of $\mathrm{p} 19^{\mathrm{ARF}}$ in tissue aging, we established a transgenic mouse (ARF-diphtheria toxin receptor [ARF-DTR]) in which the DTR (human HB-EGF I117V/L148V; ref. 14) and luciferase were expressed from the CDKN2A locus (Figure 1A). Embryonic fibroblasts prepared from these mice (MEFs) expressed p19 ${ }^{\mathrm{ARF}}, \mathrm{p} 16^{\mathrm{INK} 4 \mathrm{a}}, \mathrm{p} 21$, and luciferase when senescence was induced by either serial passages or oncogenic Ras (Figure 1, B and C). The expression of senescence markers (p19ARF and p16 ${ }^{\text {INK4a }}$ ) as well as luciferase was diminished when senescent ARF-DTR MEFs were treated with diphtheria toxin (DT) (Figure 1, D-G). An in vivo imaging analysis revealed that luciferase activity was barely detectable in young (2-month-old) female mice but became apparent in multiple tissues by the age of 12 months (Figure 2A). Similar results were obtained in male mice, with the exception of the testis (data not shown); luciferase was detectable in the testis irrespective of the age of animals, which presumably reflected the lifelong expression of $\mathrm{p} 19^{\mathrm{ARF}}$ in mouse spermatogonia (16). The luminescence signals observed in old female mice were attributed to those in the lungs and adipose tissues (Figure 2, C and D), which is consistent with the endogenous expression of $A R F$ in the lungs and adipose tissues at this age (Figure 2B). INK4a mRNA expression was also increased, as previously reported (8), confirming the accumulation of senescent cells in these tissues. However, expression of $A R F$ is increased in many tissues during aging (8), suggesting that the expression of the transgene in ARF-DTR mice does not mimic the expression pattern of endogenous $A R F$ in other tissues.

The intraperitoneal administration of DT eliminated the luminescence signal from the lungs within 48 hours (Figure 2, C and D), and this effect lasted for at least 2 weeks after drug administration (Supplemental Figure 1; supplemental material available online with this article; doi:10.1172/jci.insight.87732DS1). In contrast, the DT treatment failed to reproducibly decrease luciferase activity in adipose tissue for an unknown reason. Based on the above results, we analyzed the role of $\mathrm{p} 19^{\mathrm{ARF}}$-expressing cells in lung function using female ARF-DTR mice.

The aging-associated decline in lung function is characterized by an increase in tissue compliance due to the progressive loss of tissue elasticity, which results in the incomplete contraction of lung tissue during the expiration phase (17-19). Luciferase was not detected in bronchoalveolar lavage fluid cells but was detected in the fraction mainly containing fibroblasts (Figure 3, A and B). Immunohistochemistry revealed that endogenous p19ARF colocalized with luciferase and the senescence marker $\gamma \mathrm{H} 2 \mathrm{AX}$ in 12-month-old lung tissue (Figure 3, D and E). Luciferase was observed in cells expressing fibroblast markers (vimentin and ER-TR7) but not in those expressing the alveolar epithelial marker, SP-C (Figure 3, C, F, and G). Furthermore, when lung cells were sorted according to epithelial (CD31-; CD45-; EpCAM ${ }^{+}$) and mesenchymal

(CD31-; CD45-; Sca-1 ${ }^{+}$) markers, luciferase was detected in the mesenchymal population, suggesting that $19^{\mathrm{ARF}}$-expressing cells in the adult mouse lung are alveolar fibroblasts (Figure 3, H and I). Taken together, these results strongly indicate that cellular senescence is induced in the alveolar fibroblasts of the 12-month-old lung parenchyma.

Twelve-month-old mice were treated with PBS or DT (Figure 4A). Lung luciferase activity in 
A

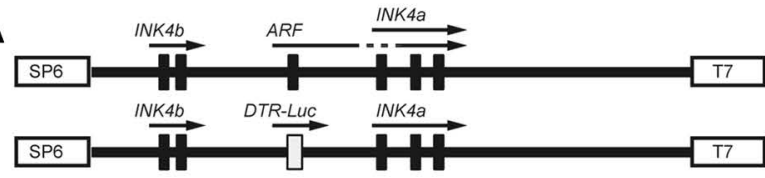

B

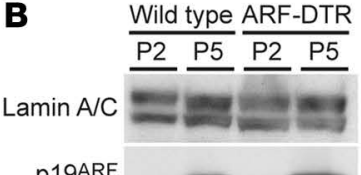

p19ARF

p16INK4a
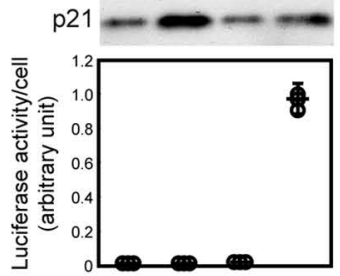

C
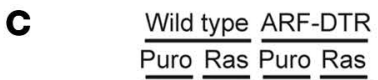

Lamin A/C

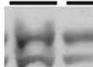

$$
\text { p19ARF }
$$

p16INK4a
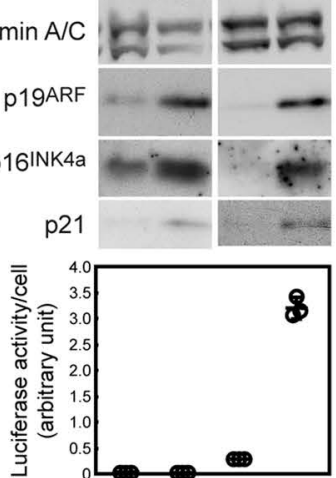

D

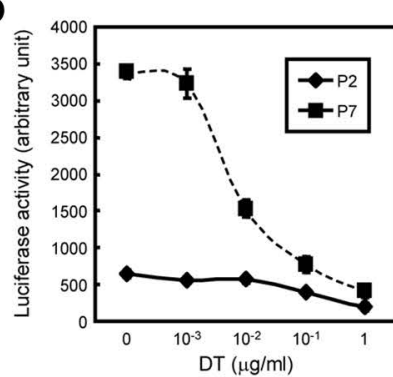

$\mathbf{F}$

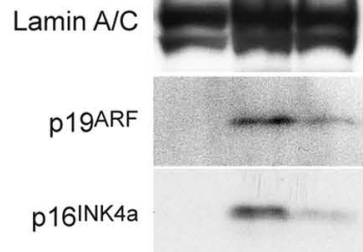

E

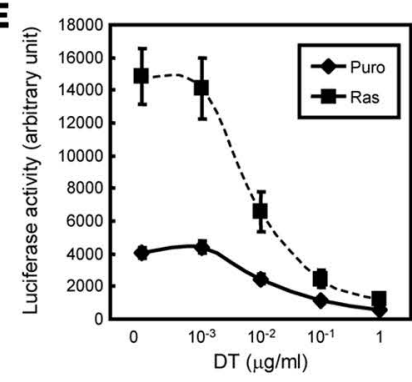

G

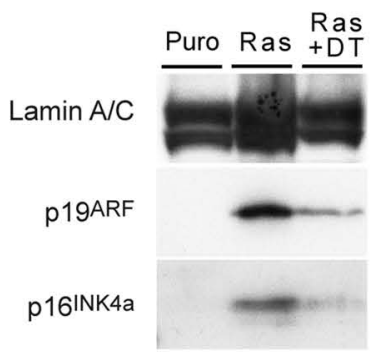

Figure 1. Establishment of ARF-DTR transgenic mice. (A) A transgenic vector was constructed using a phage artificial chromosome, including a mouse INK4a/ARF locus. ARF exon $1 \beta$ was replaced with genes encoding the diphtheria toxin receptor (DTR) (human HB-EGF I117V/L148V) fused to the self-cleaving picornavirus-derived 2A peptide sequence and firefly luciferase (DTR-Luc). (B and C) MEFs prepared from wild-type or ARF-DTR mice were cultured on a 3 T3 protocol (B) or infected with control (puro) or oncogenic RasVal12-encoding retroviruses (C) in order to induce senescence. Infected cells were selected with puromycin for 3 days. Cell lysates were prepared and luciferase activity was measured. Luciferase activity was normalized to cell numbers in each sample. Values represent the mean \pm SD of triplicate samples. Senescence was confirmed by the expression of p19ARF, p16 INK4a, and p21 in passage 5 (P5) and Ras-expressing (Val12-expressing) MEFs. Lamin A/C was used as a loading control. (D and E) Senescence was induced in ARF-DTR MEFs by serial passages (D) or oncogenic Ras (E). MEFs were then treated with the indicated concentrations of diphtheria toxin (DT) for 24 hours, and the luciferase assay was performed. Data are representative of 2 independent experiments. Values are shown as the mean \pm SD of triplicate samples. (F and $\mathbf{G}$ ) Senescence was induced in ARF-DTR MEFs by serial passages (F) or oncogenic Ras $(\mathbf{G})$. Senescent MEFs (P5 or Ras) were cultured in the absence or presence of DT $(1 \mu \mathrm{g} / \mathrm{ml})$ for 24 hours, and the expression of p19ARF and p16 $6^{\text {INK } 4 a}$ was analyzed by immunoblotting. Lamin $A / C$ was used as a loading control.

12-month-old mice decreased to a similar level as that in young mice after the DT treatment (Figure 4B). No significant changes were observed in body weights after the DT treatment (Supplemental Figure 2). The DT treatment reduced ARF, INK4a, and $p 21$ levels in the lungs of ARF-DTR mice, but not in those of wildtype mice, indicating that $\mathrm{p} 19^{\mathrm{ARF}}$-expressing senescent cells were successfully removed from lung tissue of ARF-DTR mice (Figure 4, C and D).

We then performed pulmonary function tests on these mice. As previously reported $(20,21)$, static lung compliance (Cst) was significantly higher in older animals than in young animals (Figure 5, A and B). The DT treatment resulted in the marked recovery of lung elasticity (decrease in Cst) in ARF-DTR mice but not in wild-type mice. Similarly, the DT treatment reversed aging-associated changes in dynamic compliance, dynamic resistance, tissue elastance, and tissue damping in ARF-DTR mice but not in wild-type mice (Figure 5, C-F). These results clearly indicated that the p19 $9^{\mathrm{ARF}}$-expressing cells that accumulated in 12-month-old lung tissue had deleterious effects on pulmonary function and that aging-associated declines in pulmonary function were ameliorated by the elimination of these p19 $9^{\mathrm{ARF}}$-expressing cells.

We also compared lung morphologies among these mice. An increase in alveolar size is associated with a decline in lung function during aging $(18,19,22,23)$. Alveolar mean linear intercepts (the average distance between alveolar walls that reflects the size of the alveolar space; ref. 24) were increased in 12-monthold ARF-DTR mice (Figure 6, A and B). The DT treatment reversed this phenotype but did not affect alveolar size in wild-type mice. Mean alveolar septal wall thickness was lower in 12-month-old mice than in 2-month-old mice (Figure 6C), as previously reported (25). Furthermore, the ablation of p19 ${ }^{\mathrm{ARF}}$-expressing cells fully restored the alveolar wall in ARF-DTR mice but not in wild-type mice. Increases in lung compliance in aged animals have been attributed to a decline in the number of elastic fibers, the major component of which is elastin $(19,22)$. Therefore, we performed a quantitative analysis on elastin in immunostained sections (Figure 6D). The amount of elastin in older animals was approximately half that in young animals (Figure 6E) and recovered in DT-treated ARF-DTR mice but not in wild-type mice. 
A

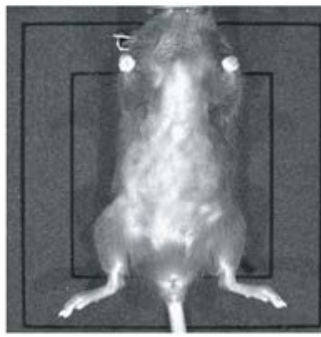

\begin{tabular}{|c|c|}
\hline $\begin{array}{c}\text { ARF-DTR } \\
2 \mathrm{mo}\end{array}$ & $\begin{array}{c}\text { ARF-DTR } \\
12 \mathrm{mo}\end{array}$ \\
\hline & $\begin{array}{c}\text { Wild type } \\
12 \mathrm{mo}\end{array}$ \\
\hline
\end{tabular}
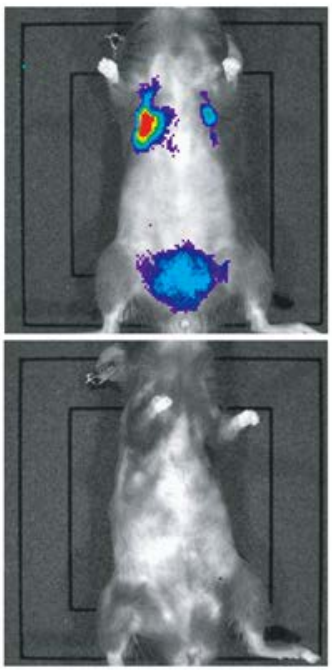

C

0

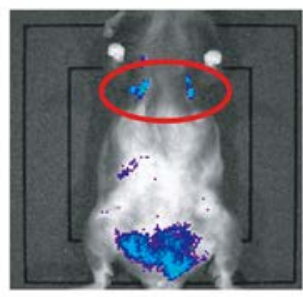

2

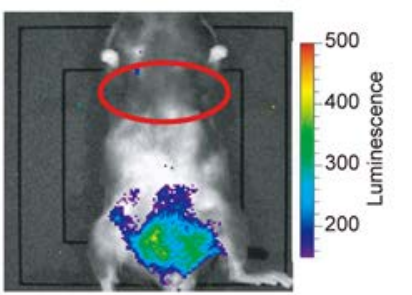

B

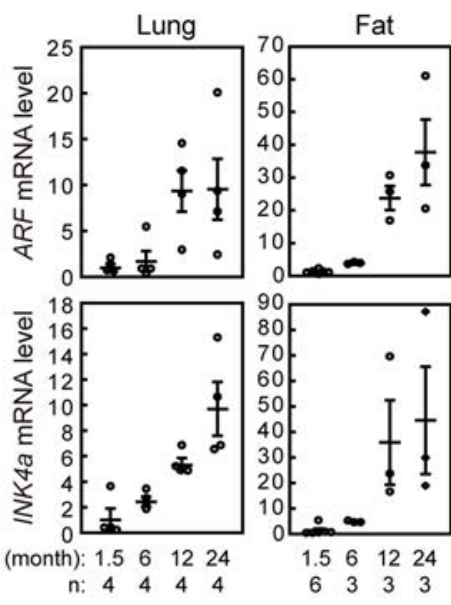

D

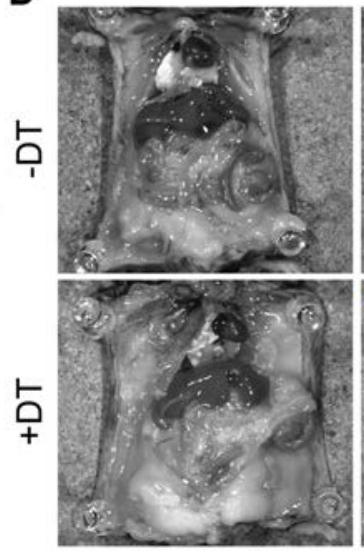

Figure 2. A luciferase signal was detected in 12-month-old ARF-DTR transgenic mice. (A) D-luciferin (150 mg/kg body weight) was injected intraperitoneally into 2- or 12-month-old female ARF-diphtheria toxin receptor (ARF-DTR) or 12-month-old female wild-type mice. Ten minutes after the injection of luciferin, an in vivo imaging analysis was performed in order to detect luminescence with a 3-minute exposure. Mice were shaved before the injection. (B) Total RNA was extracted from wild-type mouse lung and perigonadal adipose tissues at the indicated ages. ARF and INK4a mRNA levels were analyzed by real-time PCR and normalized to that of $18 \mathrm{~S}$ rRNA. Values represent mean \pm SEM of at least 3 independent experiments. (C) Luciferase activity was monitored in 12-month-old female ARF-DTR mice before (left) and 2 days (right) after an intraperitoneal injection of diphtheria toxin (DT). Signals in the red circle indicate lung tissue luciferase activity. (D) Luciferin was injected intraperitoneally into 12-month-old female ARF-DTR mice treated or untreated with DT for 3 days. Ten minutes later, mice were sacrificed and subjected to laparotomy, and in vivo luciferase activity was analyzed. A light image (left) and superimposed image (right) are shown. Lu, lung; Ad, adipose tissue. Representative images of 3 independent experiments are shown.

We also examined the effects of ARF-expressing cell elimination on even older animals. Tumor-free female ARF-DTR or wild-type mice between 20 and 22 months old were treated with PBS or DT for 1 month (Figure 7A). In vivo imaging analysis confirmed that luciferase signal disappeared from lung tissue (Figure 7B). Pulmonary function tests revealed that tissue compliance in older mice was similar to that in 12-month-old mice (Figure 7, C, D, and F). The DT treatment reduced tissue compliance in older animals; however, this effect was less than that observed in 12-month-old mice. Similarly, the DT treatment reversed aging-associated decreases in dynamic resistance and tissue damping (Figure 7, E and G). Alveolar size, which was larger than that in 12-month-old mice, was slightly restored in DT-treated animals (Figure 7, H and I). Collectively, these results indicated that $\mathrm{p} 19^{\mathrm{ARF}}$-expressing cells provoked the loss of elastic fibers in lung tissue and were also responsible for the increase in lung compliance in aged animals.

Senescent cells express cell cycle-regulating genes as well as a series of genes that function non-cell autonomously, such as cytokines, thereby affecting the behaviors of their surrounding "nonsenescent" cells $(26,27)$. In order to gain an insight into the relationship between $19^{\mathrm{ARF}}$-expressing cells and agingassociated phenotypes in lungs, we examined the expression of senescence-associated genes in these animals (Figure 8A). Among the MMPs examined, MMP-10 and -12 were significantly increased in older mice and were completely abolished or diminished in DT-treated ARF-DTR mice but not in wild-type animals (Supplemental Figure 3). These MMPs have been shown to exhibit elastase activities $(28,29)$ 
A

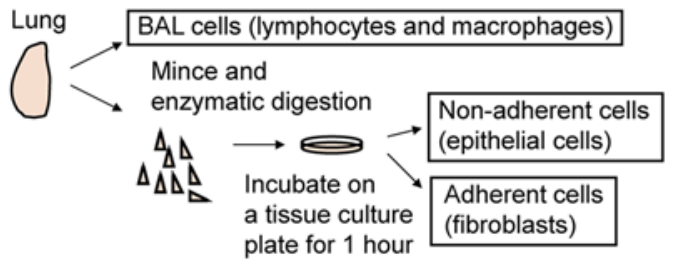

C

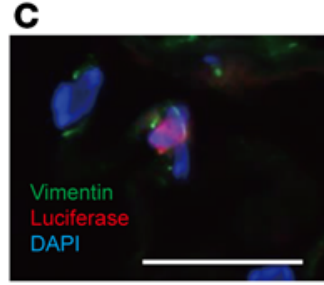

D

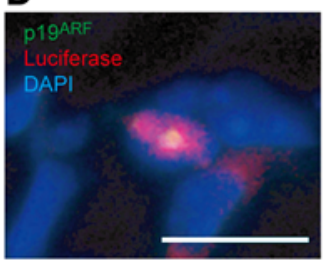

$\mathbf{F}$
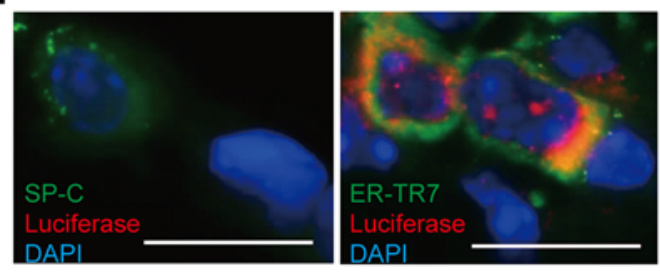

B

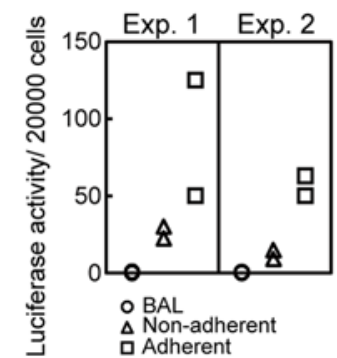

E
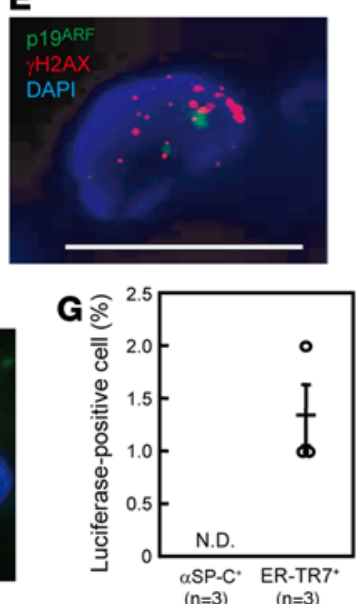

$\mathbf{H}$

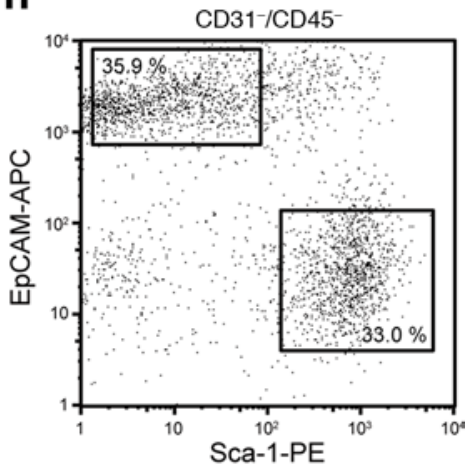

I

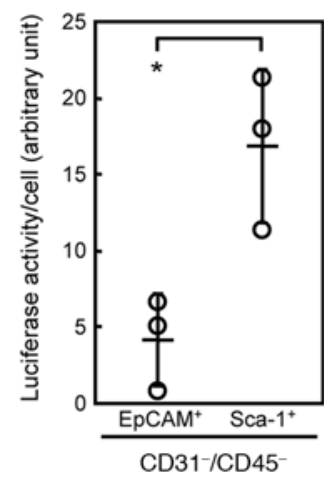

Figure 3. Luciferase was expressed in alveolar fibroblasts in old ARF-DTR mice. (A) Lung cell fractionation. Bronchoalveolar lavage (BAL) fluid mainly containing macrophages was initially obtained from the lung. The rest of the tissue was minced, trypsinized, and seeded on a tissue culture dish. After being incubated for 1 hour, floating cells that contained epithelial cells were collected. Attached cells were trypsinized to recover alveolar fibroblasts. (B) Lysates were prepared from the collected cells, and the luciferase assay was performed. Luciferase activity was normalized to the cell number in each sample. Results of 2 independent experiments are shown. (C-E) Immunofluorescence staining of the ARF-diphtheria toxin receptor (ARF-DTR) lung. Sections were stained with antibodies against the indicated proteins. Original magnification, $\times 40(\mathbf{C}$ and $\mathbf{D}), \times 100(\mathbf{E}) ; \mathrm{scale}$ bar: $20 \mu \mathrm{m}(\mathbf{C}$ and $\mathbf{D}), 10 \mu \mathrm{m}(\mathbf{E}) .(\mathbf{F})$ Immunofluorescence staining of the ARF-DTR lung. Sections were costained with surfactant-associated protein C (SP-C) or the ER-TR7 fibroblast marker together with luciferase. Sections were counterstained with DAPI. Original magnification, $\times 100$; scale bar: $10 \mu \mathrm{m}$. (C) The luciferase-positive population in SP-C- or ER-TR7-stained cells was counted. At least 100 SP-C- or ER-TR-7-positive cells were analyzed in each section. Values represent the mean \pm SEM of 3 independent experiments. N.D., not detected. (H) Gating strategy for CD31-; CD45-; EpCAM ${ }^{+}$epithelial cells and CD31-; CD45-; Sca-1+ mesenchymal cells Lung cells from 7 ARF-DTR mice were pooled before sorting. (I) Luciferase activity was determined in gated epithelial and mesenchymal cells shown in (H). Luciferase activity was normalized to cell numbers in each sample. A representative result of 2 independent experiments is shown. Values represent the mean \pm SD of triplicate samples. ${ }^{*} P<0.05$, unpaired Student's $t$ test.

and are, thus, good candidates for the reduced level of elastin observed in the lungs of aged animals. $M M P-12$ knockout mice were found to resist the degradation of elastin when emphysema was induced by cigarette smoking (30). MMP-10 protein was also downregulated in DT-treated ARF-DTR lungs but not to the same extent as their mRNA levels (Supplemental Figure 4). In order to ascertain whether increases in MMP were responsible for the reduced elastin level, MEFs were infected with retroviruses encoding shRNA against MMP-10. Cells were then subjected to ionizing radiation and cultured under 3\% oxygen to induce senescence-associated genes in MEFs (31). MMP-10 level was decreased to approximately half by shRNA (Figure 8B). Elastin levels were reduced to approximately half that of untreated cells cultured under $20 \%$ oxygen (data not shown) but recovered when MMP activities were inhibited either by shRNA or a chemical inhibitor (Figure $8 \mathrm{C}$ ). Taken together, these results suggest that the loss of elastin in senescent cells was, at least partly, attributed to increased MMP.

We also compared gene expression profiles among 2-month-old and 12-month-old (with or without DT) ARF-DTR lungs. Genes differentially expressed (more than 2-fold) between 2-month-old and 12-monthold lungs were identified (Supplemental Table 1, a full list of genes is available from the GEO database, accession GSE64754; http://www.ncbi.nlm.nih.gov/geo/query/acc.cgi?acc=GSE64754). Changes in the expression of these genes among young and old DT-treated animals were then analyzed. In order to simplify the results obtained, genes with expression levels that changed more than 2-fold in wild-type animals after the DT treatment were subtracted from the list because they exhibited "nonspecific" responses to DT. 
A

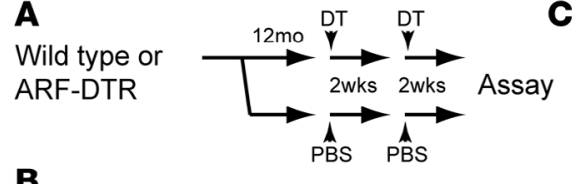

B

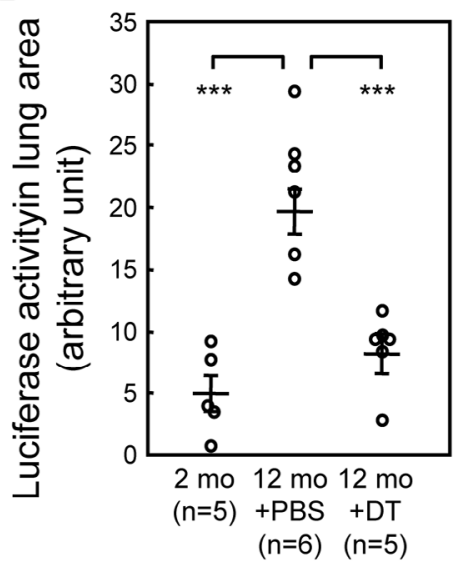

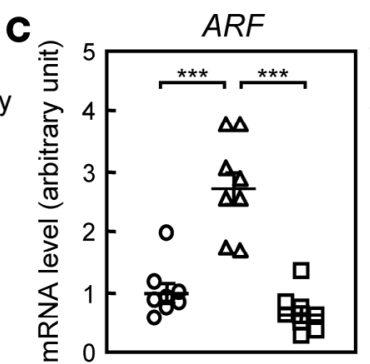
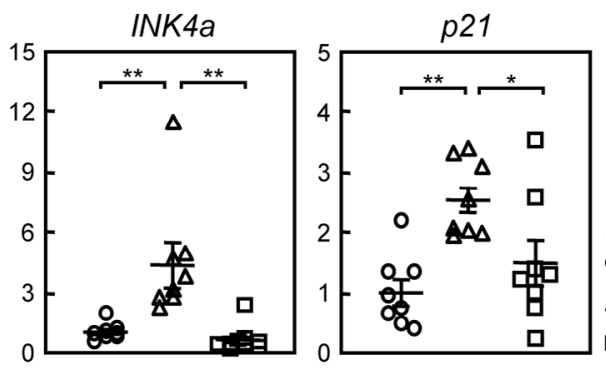

ARF-DTR

o $2 \mathrm{mo}(\mathrm{n}=8)$

$\Delta 12 \mathrm{mo}+\mathrm{PBS}(\mathrm{n}=8)$

口 $12 \mathrm{mo}+\mathrm{DT}(\mathrm{n}=8)$
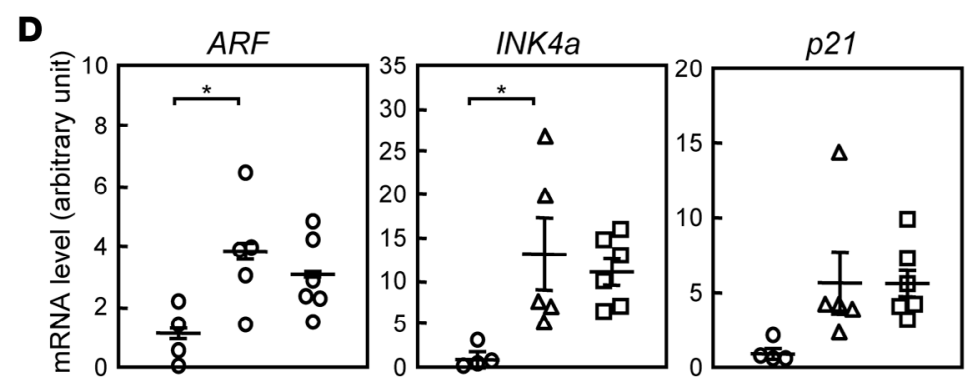

Wild type

o $2 \mathrm{mo}(\mathrm{n}=4)$

$\Delta 12 \mathrm{mo}+\mathrm{PBS}(\mathrm{n}=5)$

口 12 mo +DT $(n=6)$

Figure 4. Downregulation of senescence genes by eliminating p19ARF-expressing cells. (A) Experimental design. Twelve-month-old female wild-type or ARF-diphtheria toxin receptor (ARF-DTR) mice were intraperitoneally injected with diphtheria toxin (DT) or PBS twice with a 2-week interval. Two weeks after the second DT injection, mice were sacrificed and their lungs were analyzed. (B) Luciferase signals in the lung area were measured in ARF-DTR mice using Living Image in vivo imaging software. Values represent the mean \pm SEM of independent experiments. (C and D) Total RNA was extracted from the lungs of 2-month-old and 12-month-old female ARF-DTR mice treated with DT or not treated for 4 weeks (C) or 12-month-old wild-type mice treated or not treated with DT for 4 weeks (D). The expression of $A R F$, INK4a, and $p 21$ was analyzed by real-time PCR. mRNA levels were normalized to GAPDH in each mouse. Values represent the mean \pm SEM of independent experiments. Data were analyzed 1-way ANOVA followed by post-hoc Tukey-Kramer multiple comparison test. ${ }^{*} P<0.05,{ }^{* *} P<0.01,{ }^{* *} P<0.001$.

A pathway analysis revealed that genes involved in cytokine and chemokine signaling are differentially expressed in 12-month-old lungs, which may reflect the secretory phenotype of senescent cells $(26,27)$ (Table 1). Of the 305 genes that were upregulated in old lungs, the expression of $170(55.7 \%)$ genes was decreased following the DT treatment (Figure 9 and Supplemental Table 1). Conversely, the DT treatment upregulated the expression of $82(62.1 \%)$ of the 132 downregulated genes in old lungs. Thus, the elimination of p19 $19^{\mathrm{ARF}}$-expressing cells led to the "rejuvenation" of gene expression profiles in whole lung tissue; however, approximately $40 \%$ of genes with altered expression levels in 12-month-old animals remained intact under these conditions, suggesting that $19^{\mathrm{ARF}}$ contributed to only a certain aspect of the agingassociated phenotypes of the lung or that some aging-associated changes are irreversible.

\section{Discussion}

We established a mouse model in which it was possible to eliminate $\mathrm{p} 19^{\mathrm{ARF}}$-expressing cells using a TRECK system. By using this model, the $\mathrm{p} 19^{\mathrm{ARF}}$-expressing cells that accumulated during aging were successfully eliminated from lung tissue. Attempts to eliminate senescent cells in vivo have been made by Campisi's and van Deursen's groups $(6,13)$. The former showed that cellular senescence is induced during wound healing and promotes wound closure by enhancing myofibroblast differentiation through SASP. The latter documented the role of senescence in naturally occurring tissue aging, including the kidney and heart, and, more importantly, showed that senescent cells limited the life span of model mice. So far, we obtained only one transgenic mouse line in which ARF-DTR transgene exhibited the senescence-dependent expression. The transgene expression reflected endogenous $A R F$ expression in at least lung and adipose tissues but not in other tissue such as liver in which $A R F$ is induced during aging (8), precluding the analysis of the roles of p19 $9^{\mathrm{ARF}}$-expressing cells in other tissues or life spans. Nonetheless, our results clearly demonstrated that the elimination of $\mathrm{p} 19^{\mathrm{ARF}}$-expressing cells from lung tissue ameliorated aging-associated phenotypes, including reduced tissue elasticity, morphological changes, and gene expression. Therefore, aging-associated pulmonary hypofunction was caused, at least partly, by $\mathrm{p} 19^{\mathrm{ARF}}$, which accumulates during aging.

In our mouse model, DTR and luciferase are expressed under the control of $A R F$ promoter/enhancers. Unlike in human cells, $A R F$ clearly plays essential roles in the induction of cellular senescence in mouse 
A

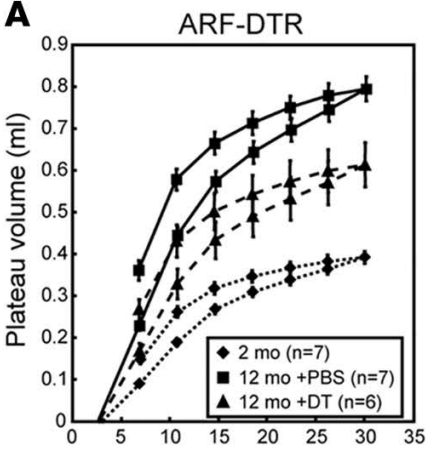

Plateau pressure $\left(\mathrm{cmH}_{2} \mathrm{O}\right)$

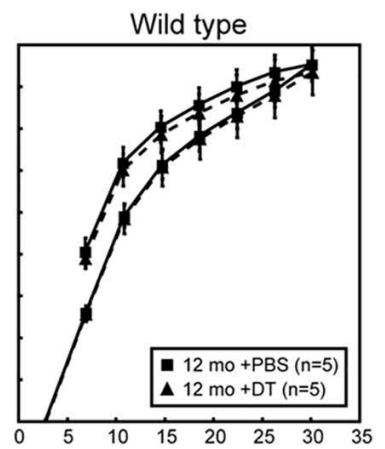

Plateau pressure $\left(\mathrm{cmH}_{2} \mathrm{O}\right)$

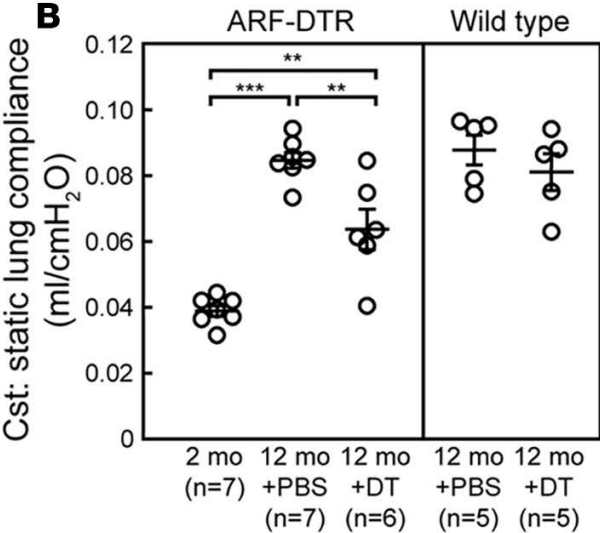

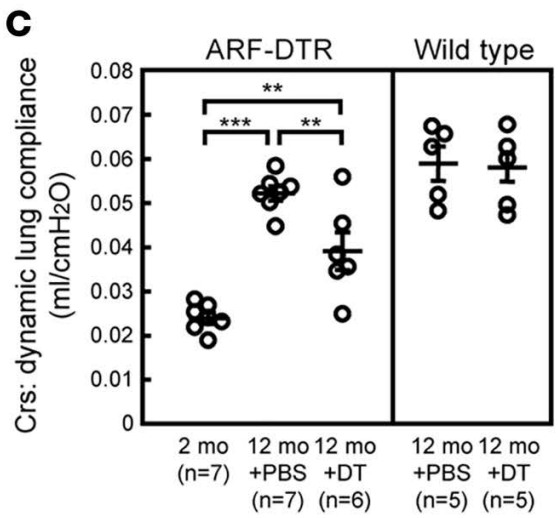
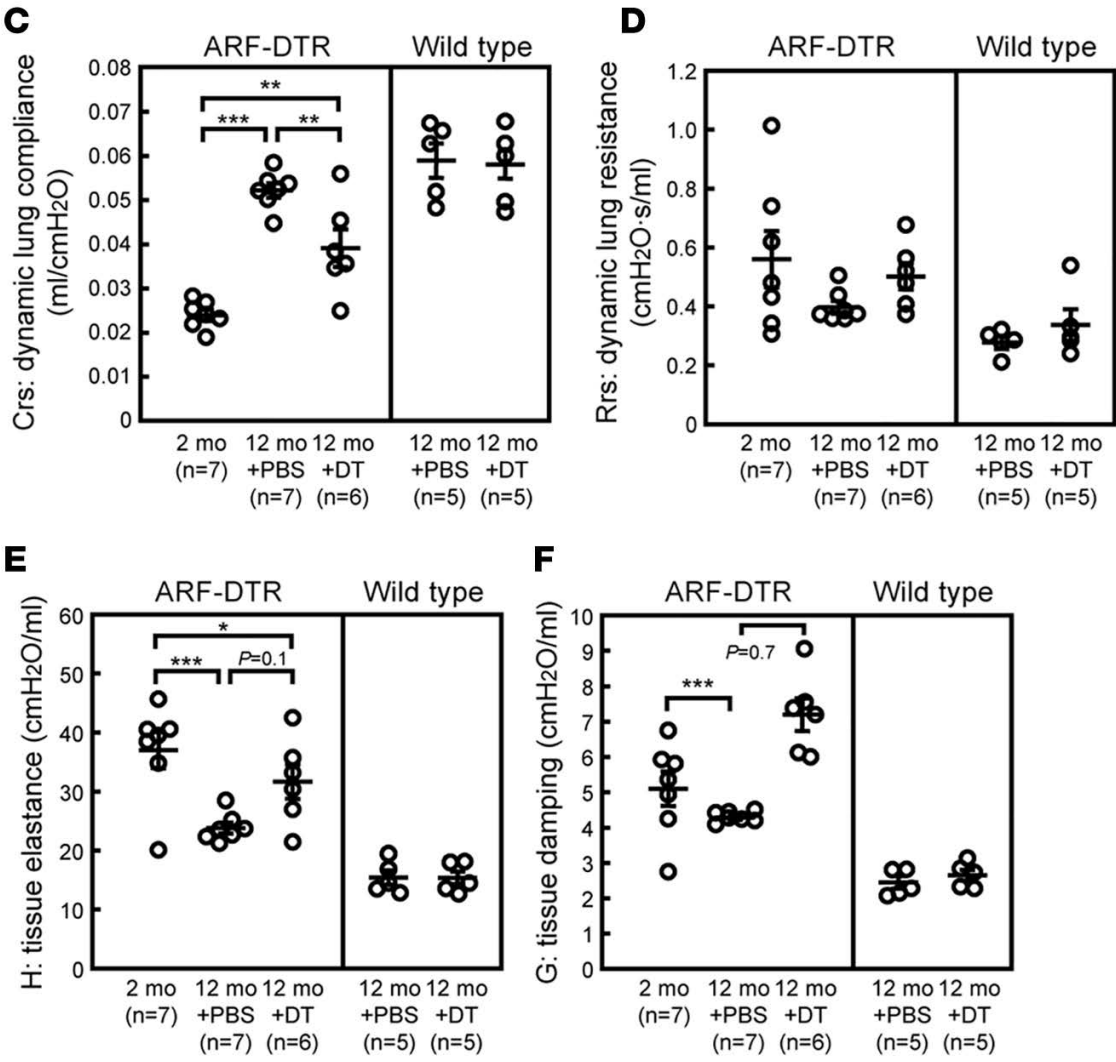

Figure 5. Restoration of lung function by eliminating p19ARF-expressing cells. (A) Pressure-volume curves of ARF-diphtheria toxin receptor (ARF-DTR) and wild-type mouse lungs treated with diphtheria toxin (DT) or PBS, as depicted in Figure 4A. Mice in the indicated groups were euthanized and connected to the FlexiVent system through their tracheae. Mice were subjected to laparotomy, and diaphragms were removed prior to the assay. (B) Static lung compliance (Cst) was calculated from the slope of the pressure-volume loop. Cst reflects the static elastic recoil pressure of the lungs at a given lung volume. (C-F) Dynamic lung compliance (Crs) (C), dynamic lung resistance (Rrs) (D), tissue elastance (H) (E), and tissue damping (C) (F) are shown. Dynamic lung compliance captures the ease with which the lungs may be extended, and dynamic lung elastance captures the elastic rigidity or stiffness of the lungs. Tissue elastance and tissue damping reflect energy conservation and dissipation in lung alveoli, respectively. Values represent mean \pm SEM of independent experiments. Data were analyzed 1-way ANOVA followed by post-hoc Tukey-Kramer multiple comparison test. ${ }^{*} P<0.05,{ }^{* *} P<0.01,{ }^{* * *} P<0.001$.

cells. p16 $6^{\mathrm{INK} 4 \mathrm{a}}$ and $\mathrm{p} 19^{\mathrm{ARF}}$ are both induced during senescence in mouse cells. Nevertheless, INK4a is largely dispensable for senescence in MEFs $(32,33)$, while $A R F$-null MEFs do not have a replicative life span and knockout mice are highly prone to cancer (34). Although we were unable to detect senescence-associated $\beta$-galactosidase activity in old lung tissue sections, $\mathrm{p} 19^{\mathrm{ARF}}$-expressing cells were positive for $\gamma \mathrm{H} 2 \mathrm{AX}$, and the elimination of these cells also blunted the expression of other senescent markers, including INK $4 a$ and $p 21$. Hence, our results strongly suggest that p19 ${ }^{\mathrm{ARF}}$-expressing cells in lung tissue represent senescent cells. However, it should be noted that $A R F$ is less important or dispensable for the senescence of human cells, since $\mathrm{p} 14^{\mathrm{ARF}}$ is not induced during senescence. Expression of $A R F$ is more limited in humans, and hyper- 
Table 1. Functional gene categories of differentially expressed genes between 2- and 12-month-old mice lung tissues

\begin{tabular}{lc}
\hline Gene set name & P value (FDR adjusted) \\
Circadian rhythm - mammal & $4.40 E-05$ \\
Tight junction & $1.76 E-04$ \\
Cytokine-cytokine receptor interaction & $1.76 E-04$ \\
\hline Chemokine signaling pathway & $9.71 E-04$ \\
Neuroactive ligand-receptor interaction & $7.35 E-03$ \\
& $3.45 E-02$ \\
Calcium signaling pathway & $4.41 E-02$ \\
Leukocyte transendothelial migration & $4.41 E-02$ \\
\hline Focal adhesion & \\
Two- and twelve-month-old female ARF-DTR mouse lungs were analyzed by microarrays (Figure 9 and Supplemental \\
Table 1), and a pathway analysis of differentially expressed genes in the lungs of 12-month-old mice was performed \\
using KEGG gene sets. Significantly enriched gene sets (FDR-adjusted P < 0.05) are listed.
\end{tabular}

physiological activation of the E2F transcription factor is required for its induction $(35,36)$. Therefore, it is unlikely that $\mathrm{p} 14^{\mathrm{ARF}}$ exhibits aging/senescence-dependent expression in human tissue.

We observed luciferase and p19ARF expression in alveolar fibroblasts. Fibroblasts are thought to be a major source of extracellular matrix and play a crucial role in the maintenance of tissue structure. Thus, changes in fibroblast function caused by $19^{\mathrm{ARF}}$ expression may directly cause a pulmonary dysfunction. However, this is unlikely, because only a small fraction of fibroblasts expressed luciferase and p19ARF. Additionally, it cannot be formally excluded that an extremely small number of epithelial or other cells also express p19 ${ }^{\mathrm{ARF}}$ and cause pulmonary aging. Further studies are required to determine whether other cells also contribute to the phenotypes observed in 12-month-old mice.

Senescent cells are known to have an effect on their surrounding "nonsenescent" cells through SASP (27). Our results suggest that the population of p19ARF-expressing cells was very small, even in adult lung tissue (approximately $1 \%$ of the lung mesenchymal population). Nevertheless, our microarray data indicate that these "rare" p19 $19^{\mathrm{ARF}}$-expressing cells strongly influence gene expression in lung tissue. Hundreds of genes are upregulated and downregulated during aging in the lung, and more than half of these aging-associated genes show p19 ${ }^{A R F}$ dependence. Since senescent cells non-cell autonomously induce senescence-like gene expression in their surrounding cells through SASP (37), it is reasonable to assume that changes in aging-associated genes in lung tissue do not simply reflect the events within p19 ${ }^{\mathrm{ARF}}$-expressing cells, but also include global changes in lung tissue cells that are mostly nonsenescent. A pathway analysis revealed that cytokine and chemokine signaling pathways are differentially regulated in 2-month-old and 12-month-old lung tissues, which is expected since most SASP genes participate in these signaling pathways (38). In addition to cytokine and chemokine signaling pathways, our results suggest that genes involved in circadian rhythms are also differentially regulated in older lung tissues. Previous studies demonstrated that the circadian clock affected pulmonary inflammation $(39,40)$. Thus, aging may affect lung pathology by modulating circadian clock-dependent gene expression.

Aging is considered to underlie many diseases in humans, and this is also the case in the lungs because the risk of chronic obstructive pulmonary disease (COPD) increases with age and is currently one of the main causes of death worldwide. Although aging-associated changes in lung tissue may not directly cause pulmonary diseases, aging facilitates the development of cigarette smoke-induced inflammation and emphysema $(41,42)$. Cigarette smoking has been shown to markedly increase the incidence of COPD in humans (43). The number of senescent cells also increases under these circumstances $(44,45)$. Hence, these findings, taken together with our results, suggest a possibility that emphysema is prevented by suppressing cellular senescence. Furthermore, senescent cells accumulate not only in the lungs, but also in other tissues, and are considered to be responsible for several aging-associated phenotypes and diseases $(9,13,46,47)$. Future studies will clarify the role of senescent cells in the aging of other organs and the availability of a senescent cell-targeted therapeutic approach, such as senolytic agents (48-50), for aging-associated diseases. 
A
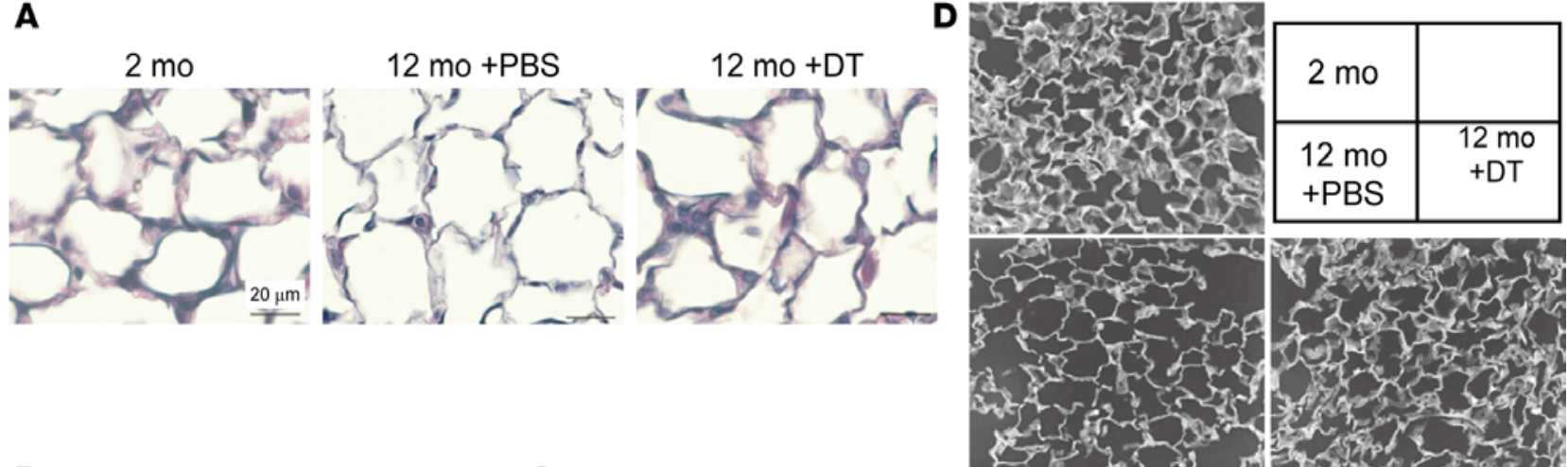

B

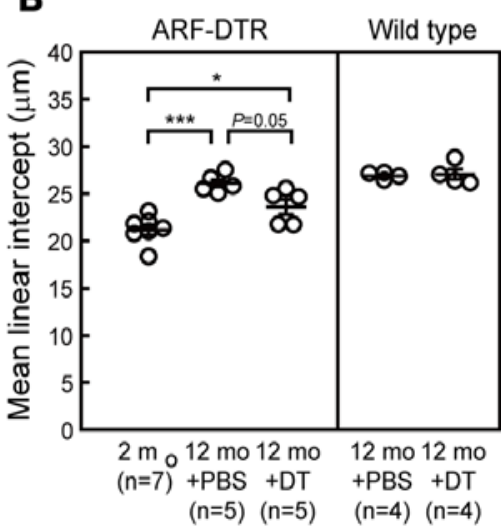

C

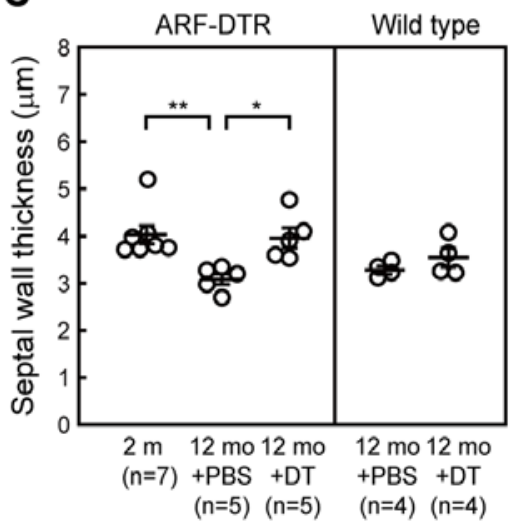

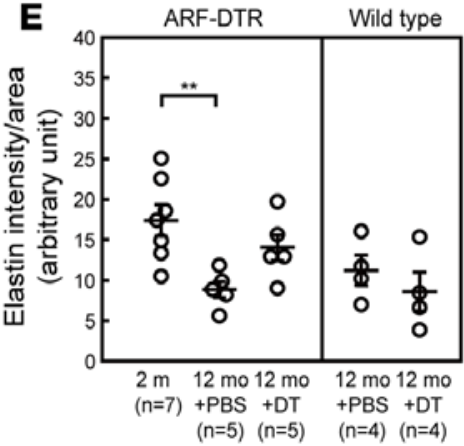

Figure 6. Effects of p19ARF-expressing cell elimination on lung morphology. (A) Mice were treated with diphtheria toxin (DT) or PBS, as depicted in Figure 4A. Lung tissues were fixed with Bouin solution at $25 \mathrm{cmH}_{2} \mathrm{O}$ and subjected to Resorcin-Fuchsin staining, followed by counterstaining with hematoxylin-eosin. Original magnification, $\times 120$ (using digital zoom); scale bar: $20 \mu \mathrm{m}$. (B and C) Alveolar mean linear intercepts (B) and septal wall thickness (C) were measured. More than 400 alveoli and 150 septal walls per mouse were counted. (D) Images of the elastin immunostaining of lung sections. Lung sections were immunostained with anti-elastin and visualized using a HRP-conjugated secondary antibody. Immunostaining in each sample was performed under identical conditions. Original magnification, $\times 40$. (E) Captured elastin immunostaining images were analyzed by Image for the quantitation of elastin signals per field. Values represent the mean \pm SEM of independent experiments. Data were analyzed 1-way ANOVA followed by post-hoc Tukey-Kramer multiple comparison test. ${ }^{*} P<0.05,{ }^{* *} P<0.01,{ }^{* *} P<0.001$.

\section{Methods}

PAC transgenic construct. A mouse $A R F / I N K 4 a / I N K 4 b$ PAC clone was provided by Gordon Peters (Cancer Research UK, London, United Kingdom). The PAC clone contained the complete $19 \mathrm{~kb}$ of the mouse $A R F$ genomic sequence, with an additional $25 \mathrm{~kb}$ of the $5^{\prime}$ - and $33 \mathrm{~kb}$ of the $3^{\prime}$-flanking genomic regions of the mouse $A R F$ gene, respectively (Figure 1A). A chimeric $A R F$-DTR-2A-luc PAC transgenic construct that contains a tandem cassette of the human heparin-binding epidermal growth factor-like growth factor (HB-EGF) with the DTR-generating I117V/L148V mutation (14), the 2A peptide gene, and a luciferase reporter gene was derived from the pGL3 Basic vector (Promega) in place of the start codon of the $A R F$ gene locus, was generated by recombineering (Figure 1A). The DTR-2A-luc gene was transferred to the $A R F$ PAC clone using the Red/ET Counter Selection BAC Modification Kit (Gene Bridges) (51). In brief, a rpsL-neo counter selection cassette flanked by two adjacent sequences of the start codon of the $A R F$ gene was amplified by PCR. The amplified rpsL-neo counter selection cassette was inserted into the $A R F$ gene of the PAC clone by Red/ET recombination. The DTR-2A-luc gene was subcloned to plasmid pENTR1A (Thermo Fisher Scientific). The subcloned DTR-2A-luc fragment was flanked by 30 nucleotide sequences of the $A R F$ gene untranslated region and the exon sequence directly adjacent to the start codon. The DTR2A-luc gene fragment was precisely transferred to the $A R F$ gene of the mouse PAC clone by Red/ET recombination in order to construct a chimeric $A R F$-DTR-2A-luc PAC clone. The PAC modification was verified by sequencing. The $A R F$-DTR-2A-luc PAC transgenic construct was purified for a microinjection with a slightly modified procedure (52). The PAC transgenic construct was extracted from $250 \mathrm{ml}$ of the E. coli culture using the Nucleobond Plasmid Purification kit (MACHEREY-NAGEL). In order to achieve purification, $10 \mu \mathrm{g}$ of the PAC transgenic construct was linearized overnight with SfiI (New England Bio- 
A

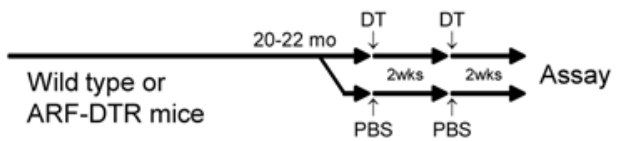

B
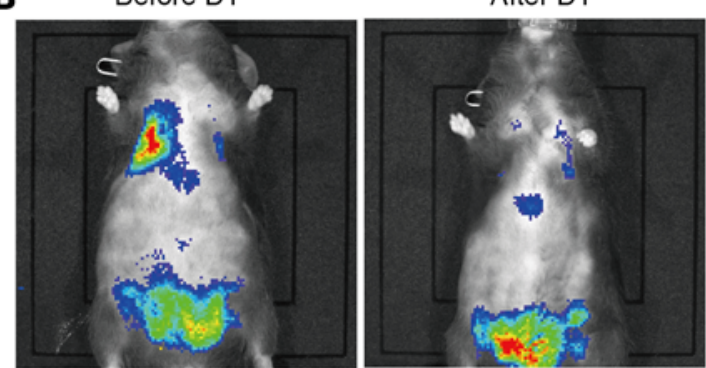

C
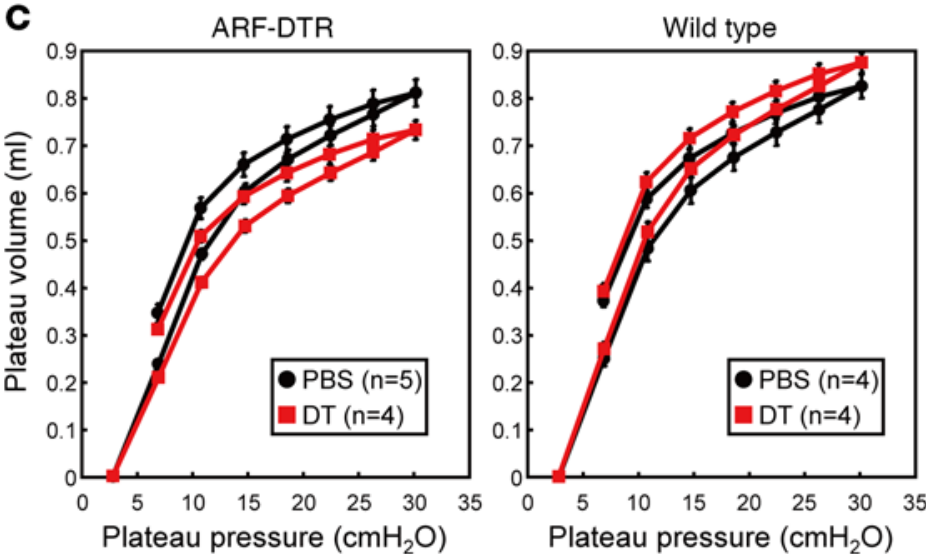

D

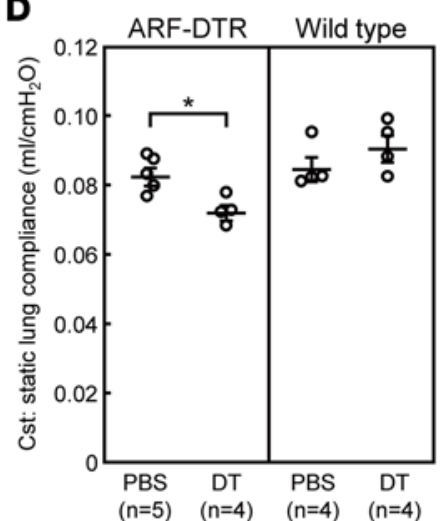

E

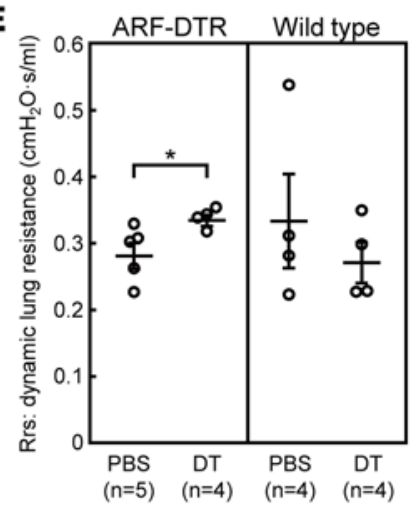

$\mathbf{F}$

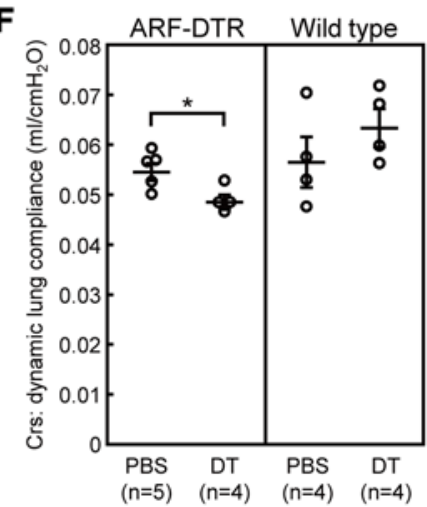

H

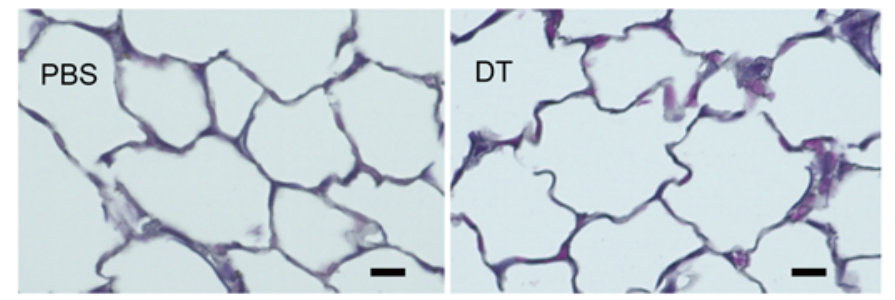

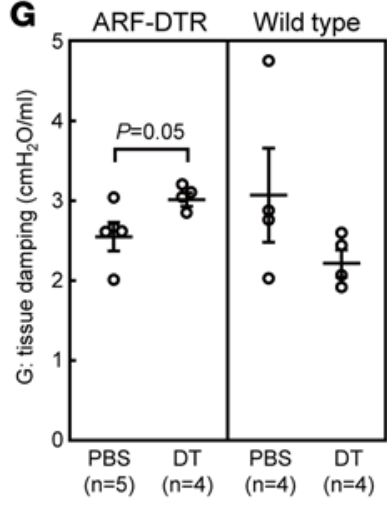

I

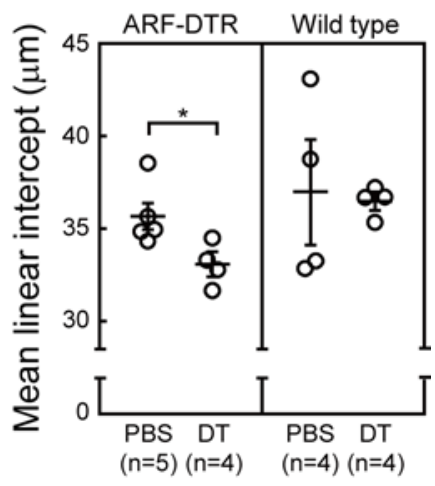

Figure 7. Effects of the DT treatment on older mice. (A) Experimental design. Twenty- to twenty-two-month-old female ARF-diphtheria toxin receptor (ARF-

DTR) or wild-type mice were intraperitoneally injected with diphtheria toxin (DT) or PBS twice with a 2-week interval. Two weeks after the second DT injection, mice were sacrificed and their lungs were analyzed. (B) Luciferase activity was monitored in ARF-DTR mice before (Pre-DT) and after (Post-DT) DT treatment. (C) Pressurevolume curves of mouse lungs treated with DT or PBS. (D-C) Static lung compliance (Cst), dynamic resistance (Rrs), dynamic compliance (Crs), and tissue damping (G). (H) Images of the lungs of PBS-and DT-treated mice. Sections were subjected to Resorcin-Fuchsin staining followed by counterstaining with hematoxylin-eosin staining. Original magnification, $\times 120$ (using digital zoom); scale bar: $10 \mu \mathrm{m}$. (I) Alveolar mean linear intercepts of PBS-and DT-treated mice were shown.

Values represent mean \pm SEM of independent experiments. ${ }^{*} P<0.05$, unpaired Student's $t$ test.

labs), cleaving a unique site in the PAC vector sequence. Linearized PAC DNA was separated with pulsed field gel electrophoresis (PFGE) and extracted from the preparative pulsed field gel by electroelution. After dialysis against TE buffer containing $0.1 \mathrm{mM}$ EDTA, aliquots were subjected to PFGE for size and quality control. The PAC DNA concentration was adjusted to $1 \mathrm{ng} / \mu 1$ for the microinjection. Aliquots of the PAC DNA solution were stored at $4^{\circ} \mathrm{C}$ for later microinjection.

ARF-DTR transgenic mice. ARF-DTR mice were generated by a pronuclear injection of C57BL/6J mouse 
A
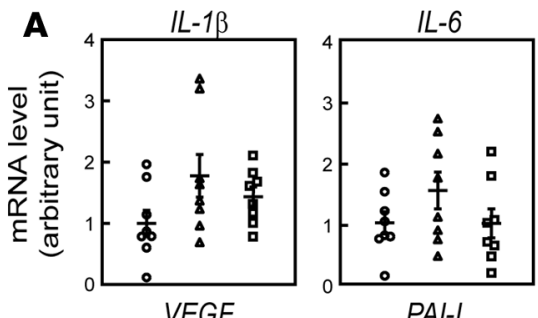

PAI-I
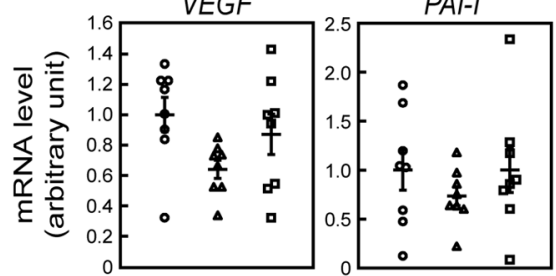

- $2 \mathrm{mo}(\mathrm{n}=8)$

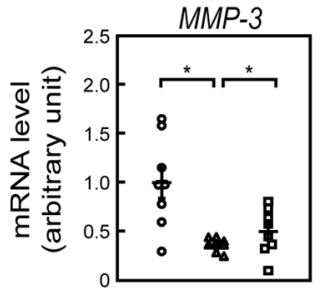

MMP-10

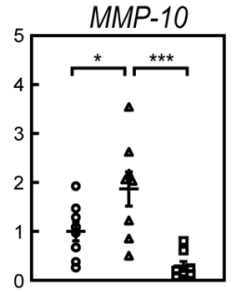

$\triangle 12 \mathrm{mo}+\mathrm{PBS}$ $(n=8)$

व $12 \mathrm{mo}+\mathrm{DT}$ $(n=8)$

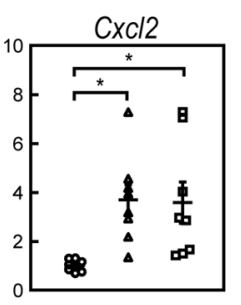

MMP-12

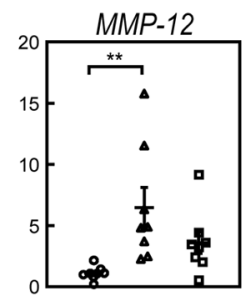

B

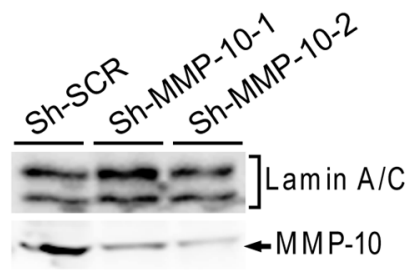

C

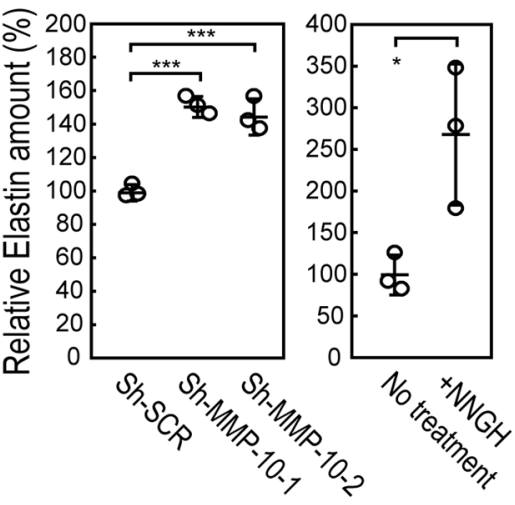

Figure 8. Changes in senescence-associated gene expression in DT-treated lungs. (A) Total RNA was extracted from 2and 12-month-old female ARF-diphtheria toxin receptor (ARF-DTR) mouse lung tissues. Twelve-month-old mice were treated with PBS or diphtheria toxin (DT), as depicted in Figure 4A. The expression of the indicated genes was analyzed by real-time PCR. mRNA levels were normalized to GAPDH mRNA in each sample. Values represent the mean $\pm \mathrm{SEM}$. (B) Wild-type MEFs were infected with retroviruses encoding shRNA against MMP-10. Two-independent shRNA were used to knockdown MMP-10. Scrambled short hairpin (sh-SCR) was used as a control in each group. Infected cells were selected with puromycin for 3 days, exposed to ionizing radiation (10 Gy), and cultured under $3 \%$ oxygen conditions for 10 days. The expression of MMP-10 was analyzed by immunoblotting. Lamin A/C was used as a loading control. (C) shRNA-infected senescent MEFs were analyzed for the amount of elastin. In the chemical inhibition of MMPs, senescent MEFs were cultured in the presence of an MMP inhibitor [ $\mathrm{N}$-isobutyl-N-(4-methoxyphenylsulfonyl)-glycylhydroxamic acid $\{\mathrm{NNGH}\}]$. Media were replaced every 3 days. The amount of elastin was measured and then normalized to the total protein content in each sample. Values represent the mean \pm SD of triplicate samples. Data are representative of 2 independent experiments. Data were analyzed 1-way ANOVA followed by post-hoc Tukey-Kramer multiple comparison test (for comparison of 3 groups) or unpaired Student's $t$ test. ${ }^{*} P<0.05,{ }^{* *} P<0.01,{ }^{* *} P<0.001$.

embryos (CLEA Japan Inc.). The transgenic founders of the PAC transgenic construct were assessed by Southern blotting of HincI-digested tail DNA probed by the $\left[{ }^{32} \mathrm{P}\right]$-labeled luciferase gene fragment. Fifteen of eightythree progeny $(18 \%)$ contained the transgene, as detected by the Southern blotting of tail DNA. No obvious gross phenotypic differences were apparent when transgene-positive and -negative littermates were compared. Fifteen independent transgenic founder mice were bred with C57BL/6J mice. Genomic DNA isolated from each founder mouse was analyzed by Southern blotting, and 9 founder mice carried more than 1 copy of transgene per genome. Embryonic fibroblasts were prepared form these mice, and luciferase activity was analyzed after the induction of senescence. One transgenic line that carried 10 copies of transgene per genome showed senescence-dependent luciferase expression, which was used for all analyses in this study. Regarding the DT treatment, mice were intraperitoneally injected with DT ( $50 \mu \mathrm{g} / \mathrm{kg}$ bodyweight) twice with a 2 -week interval. Mice developing tumors were excluded from all analyses. Animals were randomly assigned to groups.

Cell culture, luciferase assay, and in vitro elastin assay. MEFs were cultured using a 3T3 protocol and infected with a retrovirus as previously described (53). Oncogenic Ras (V12) retrovirus plasmid was provided by Manuel Serrano (Spanish National Cancer Research Center, Madrid, Spain). Infected cells were selected with puromycin. The luciferase assay was performed using the Luciferase Assay System (Promega). Luciferase activity was normalized to the cell number in each sample.

In order to quantitate the amount of elastin, early-passage MEFs were infected with a retrovirus expressing shRNA targeting MMP-10 or scramble shRNA. The target sequences of sh-MMP-10 were ATGGAAACCAGGTGCTAGA (sh-MMP-10-1) and GCGCAGCAGATGTCTTTCA (sh-MMP-10-2). Infected cells were cultured for 10 days under $3 \%$ oxygen conditions following exposure to ionizing radiation (10 Gy) in order 

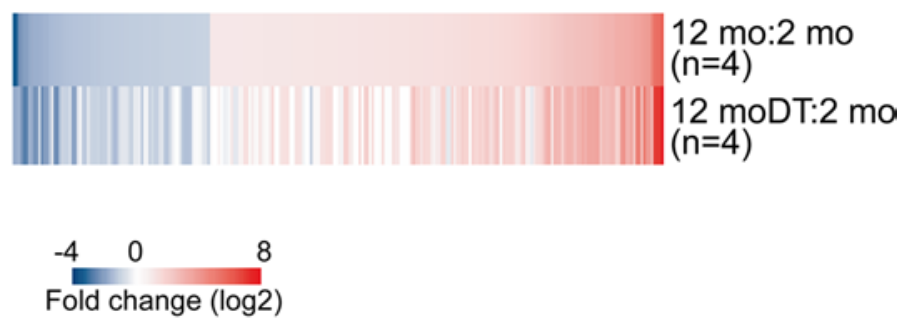

Figure 9. Effect of p19ARF-expressing cell elimination on aging-associated gene expression in lung tissue. Twelve-monthold mice were treated with PBS or diphtheria toxin (DT) for 4 weeks, as depicted in Figure 4A, before the analysis. Four mice were independently analyzed in each group, and average values were used to draw heatmaps. Cenes that nonspecifically responded to DT were subtracted from the list. The genes used to draw the heatmap are listed in Supplemental Table 1. A full list of genes is available from the GEO database (accession GSE64754). ${ }^{*} P<0.05,{ }^{* *} P<0.01,{ }^{* * *} P<0.001$

to induce SASP (31). Where indicated, $50 \mu \mathrm{M}$ of N-isobutyl-N-(4-methoxyphenylsulfonyl)-glycylhydroxamic acid (NNGH) was added to the culture medium. Media were replaced with fresh media every 3 days. The amount of elastin was determined using the Fastin Elastin Assay kit (Biocolor) and normalized to total protein.

In vivo imaging analysis. An in vivo luciferase imaging analysis was performed using the IVIS imaging system (Perkin Elmer). Mice were ventrally shaved, anesthetized with isoflurane (Wako Pure Chemicals Industries), and intraperitoneally injected with luciferin according to the manufacturer's instructions (VivoGlo; Promega). Luciferase activity was monitored 10 minutes after the luciferin injection. In order to quantify luciferase activity, signals were analyzed using Living Image software (Perkin Elmer).

Pulmonary function test. Pulmonary function tests were performed using the FlexiVent system (Scireq) as previously described $(54,55)$. Mice were euthanized by an intraperitoneal injection of pentobarbital sodium (100 mg/kg of body weight) and connected to the FlexiVent system after tracheotomy. Mice were ventilated at a respiratory rate of 150 breaths per minute, with a tidal volume of $10 \mathrm{ml} / \mathrm{kg}$ against a positive end expiratory pressure of $3 \mathrm{cmH}_{2} \mathrm{O}$. Deep inflation, Snapshot-150, Quickprime-3, and a pressure-volume loop with constant increasing pressure were consecutively performed 3 times in each subject. Cst values were calculated by fitting the Salazar-Knowles equation to the pressure-volume loop. Dynamic lung compliance and resistance values were calculated using a single frequency forced oscillation technique. Pressure, flow, and volume signals obtained from the response to a sinusoidal waveform were fit to the single compartment model using linear regression. Tissue elastance and damping, which are closely related to tissue resistance and elastance, respectively, were obtained from respiratory system impedance data using a constant phase model. All parameters were calculated using FlexiVent software.

Lung morphometry and immunohistochemistry. All histopathological analyses were performed in a blinded manner. Lungs were fixed with Bouin solution or Mildform $20 \mathrm{~N}$ (Wako Pure Chemicals Industries) at 25 $\mathrm{cmH}_{2} \mathrm{O}$. Paraffin-embedded tissues were sectioned ( $5 \mu \mathrm{m}$ thick), stained with Maeda's Resorcin-Fuchsin solution (Muto Pure Chemicals), and counterstained with hematoxylin-eosin. At least 8 randomly selected fields per mouse were photographed, and the mean linear intercepts and alveolar wall thicknesses were calculated. In the measurement of mean linear intercepts, test lines were randomly drawn on images, and the length between two sequential intersections of the alveolar surface with the test line was measured. Airway and vascular structures were eliminated from the analysis.

Immunostaining was carried out after antigen retrieval using LAB solution (Polysciences Inc.) with an elastin antibody (Abcam). Immunostained sections were mounted without counterstaining, and 4 fields not containing airway or vascular structures per mouse were photographed under equal conditions. Elastin signal intensities were analyzed using ImageJ (NIH).

In the immunofluorescence analysis, sections fixed with Bouin solution or formalin were immunostained using a p19 ARF antibody (1:50 dilution, 5-C3-1, Santa Cruz Biotechnology), surfactant-associated protein C (1:50 dilution, SP-C, M-20, Santa Cruz Biotechnology), fibroblast marker antibody (1:50 dilution, ER-TR7, Santa Cruz Biotechnology), luciferase antibody (1:100 dilution, PM016, MBL, Japan), $\gamma$ H2AX antibody (1:400 dilution, ab2893, Abcam), and vimentin antibody (1:100 dilution, RV202, Abcam). Sections were visualized with Alexa Fluor 488-conjugated anti-rat IgG (1:2000 dilution, 712-545-153, Jackson ImmunoResearch), Alexa Fluor 488-conjugated anti-goat IgG (1:2000 dilution, 705-545-147, Jackson ImmunoResearch), Alexa Fluor 488-conjugated anti-mouse IgG (1:2000 dilution, 715-545-151, Jackson 
ImmunoResearch), and Alexa594-conjugated anti-rabbit IgG (1:2000 dilution, ab150080, Abcam). Sections were counterstained with DAPI.

Immunoblotting. Lysates were prepared using RIPA buffer (10 mM Na-phosphate, pH 7.2, $150 \mathrm{mM}$ $\mathrm{NaCl}, 2 \mathrm{mM}$ EDTA, 0.1\% SDS, 1\% Na-deoxycholate, and 1\% NP-40) containing protease inhibitors. Proteins were separated by SDS-PAGE and transferred to PVDF membranes (Merck-Millipore Billerica). Proteins were detected with antibodies to p19 ${ }^{\mathrm{ARF}}, \mathrm{p} 16^{\mathrm{INK} 4 \mathrm{a}}, \mathrm{p} 21$, Lamin A/C, and $\alpha$-Tubulin (5-C3-1, M-156, F-5, H-110, and B-7; all from Santa Cruz Biotechnology); MMP-10 (bs-1344R, Bioss Inc., Boston MA); and MMP-12 (EP1261Y, Abcam). Antibodies were diluted to 1:500 (p19 ${ }^{\mathrm{ARF}}, \mathrm{p} 16^{\mathrm{INK} 4 \mathrm{a}}, \mathrm{p} 21$, Lamin A/C, $\alpha$-Tubulin, MMP-10, and MMP-12) or 1:2000 (Lamin A/C) with a blocking buffer (tris-buffered saline containing $5 \%$ skim milk and $0.1 \%$ Tween 20 ).

Real-time PCR analysis. A real-time PCR analysis was performed as previously described (56). Briefly, total RNA was reverse transcribed using the PrimeScript RT reagent kit with gDNA Eraser (Takara BIO Inc.) and subjected to PCR using the following primers: for ARF, 5'-GCCGCACCGGAATCCT-3' (sense) and 5'-TTGAGCAGAAGAGCTGCTACGT-3' (antisense); INK4a, 5'-CCCAACGCCCCGAACT-3' (sense) and 5'-GCAGAAGAGCTGCTACGTGAA-3' (antisense); IL-1 $\beta$, 5'-GAATGCCACCTTTTGACAGTG-3' (sense) and 5'-CTGGATGCTCTCATCAGGACA-3' (antisense); VEGF 5'-GCACATAGGAGAGATGAGCTTCC-3' (sense) and 5'-TTCCTGCAGCCTGGCTCACCG-3' (antisense); IL-6, 5'-CAAGAAAGACAAAGCCAGAGTC-3' (sense) and 5'-GAAATTGGGGTAGGAAGGAC-3' (antisense); PAI-1， 5'-TCAGAGCAACAAGTTCAACTACACTGAG-3' (sense) and 5'-CCCACTGTCAAGGCTCCATCACTTGCCCCA-3' (antisense); Cxcl2, 5'-CCAACCACCAGGCTACAGG-3' (sense) and 5'-GCGTCACACTCAAGCTCTG-3' (antisense); MMP-3, 5'-ACATGGAGACTTTGTCCCTTTTG-3' (sense) and 5'-TTGGCTGAGTGGTAGAGTCCC-3' (antisense); MMP-10, 5'-GAGCCACTAGCCATCCTGG-3' (sense) and 5'-CTGAGCAAGATCCATGCTTGG-3' (antisense); MMP-12, 5'-CTGCTCCCATGAATGACAGTG-3' (sense) and 5'-AGTTGCTTCTAGCCAAAGAAC-3' (antisense); GAPDH, 5'-AATGGTGAAGGTCGGTGTG-3' (sense) and 5'-GAAGATGGTGATGGGCTTCC-3' (antisense); p21, 5'-CGAGAACGGTGGAACTTTGAC-3' and 5'-CAGGGCTCAGGTAGACCTTG-3' (antisense); and 18S rRNA, 5'-AGTCCCTGCCCTTTGTACACA-3' (sense) and 5'-GATCCGAGGGCCTCACTAAAC-3' (antisense).

Cell sorting. Epithelial and mesenchymal cell separation was performed as previously reported (57). Lungs were dissected from wild-type or ARF-DTR mice, and tracheae and extrapulmonary airways were removed. Lungs were then minced using a razor blade, suspended in PBS, and incubated in $5 \mathrm{mg} / \mathrm{ml}$ of Disparse II (Roche Applied Science) at $37^{\circ} \mathrm{C}$ for 60 minutes with gentle shaking. Cells were disaggregated with an 18-gauge needle, filtered through a 50- $\mu$ m nylon cell strainer (Sysmex), and washed twice with PBS containing $2 \%$ fetal bovine serum. Low density cells were isolated by discontinuous density gradient centrifugation using Nycoprep (Nycomed Pharma) in order to remove contaminating erythrocytes, neutrophils, and cell debris. Cells were negatively selected using magnetic bead-conjugated antiCD31 (1:10 dilution, 130-097-418, Miltenyi Biotec) and -CD45 (1:10 dilution, 132-052-301, Miltenyi Biotec). Selected cells were incubated with Alexa Fluor 488-conjugated anti-mouse CD31 (1:50 dilution, 102513, BioLegend), APC-Cy7-conjugated anti-mouse CD45 (1:200 dilution, 103115, BioLegend), Alexa Fluor 647-conjugated anti-mouse EpCAM (CD326) (1:200 dilution, 118211, BioLegend), and Pacific Blue-conjugated anti-mouse Sca-1 (1:200 dilution, 108119, BioLegend). Sorting was performed using BD FACSAria II (BD Biosciences).

Microarray. Four ARF-DTR mice per group and two wild-type mice per group were independently analyzed. RNA was extracted from lung tissue using the RNeasy mini kit (Qiagen) according to the manufacturer's instructions. RNA quality and quantity were determined using the Agilent 2100 Bioanalyzer (Agilent Technologies) and Nanodrop ND1000 (Thermo Scientific). An RNA probe was prepared using the Low Input Quick Amp Labeling Kit (Agilent Technologies). Labeled RNA was hybridized to the SurePrint G3 Mouse Gene Expression $8 \times 60 \mathrm{~K}$ Microarray (Agilent Technologies) and scanned by the Agilent DNA microarray Scanner (G2539a) using Scan Control (version A.8.5.1). The scanned images were analyzed with Agilent Feature Extraction (version 10.10.1.1). Full microarray data and protocol details are available from the GEO database, accession GSE64754; http://www.ncbi.nlm.nih.gov/geo/query/acc.cgi?acc=GSE64754).

A pathway analysis was performed using KEGG (Kyoto Encyclopedia of Genes and Genomes) (58, 59) gene sets with Molecular Signatures Database program version 5.0 (http://software.broadinstitute.org/ gsea/msigdb/index.jsp). 
Statistics. For the comparison of 3 sets of data, 1-way ANOVA was performed. When the statistical model was proven significant, the differences between combinations of the 2 groups were analyzed using a Tukey-Kramer test. For the comparison of 2 sets of experimental data, a 2-tailed unpaired Student's $t$ test was used. Data displayed normal variance. $P$ values of less that 0.05 were considered significant. No blinding was performed, except for histological quantifications. No statistical method was used to determine sample size.

Study approval. All animal experiments were approved by and conducted in accordance with guidelines established by the National Center for Geriatrics and Gerontology Animal Ethics Committee.

\section{Author contributions}

$\mathrm{MH}, \mathrm{AA}, \mathrm{HK}, \mathrm{RM}, \mathrm{KK}$, and KS designed and performed experiments. YI performed microarray analysis. $\mathrm{MM}$ and TS interpreted data. MS designed experiments and wrote the paper.

\section{Acknowledgments}

We appreciate Yoshinori Naoe for his technical assistance with cell sorting and Noboru Motoyama for his valuable discussions and comments. We also thank Noboru Ogiso for his advice on animal experiments. The mouse PAC clone and Ras (Val12) retrovirus plasmid were provided by Gordon Peters and Manuel Serrano, respectively. This project was supported by grants from the National Center for Geriatrics and Gerontology (22-19, 25-18, and 28-22), the Japan Society for the Promotion of Science (KAKENHI 16K08604), the Japan Foundation for Aging and Health, and the Aichi Health Promotion Foundation.

Address correspondence to: Masataka Sugimoto, Research Institute, Department of Mechanism of Aging, National Center for Geriatrics and Gerontology, Morioka-cho, Obu, Aichi 474-8511, Japan. Phone: 81.562.46.2311; E-mail: msugimot@ncgg.go.jp.

1. Hayflick L, Moorhead PS. The serial cultivation of human diploid cell strains. Exp Cell Res. 1961;25:585-621.

2. Ben-Porath I, Weinberg RA. The signals and pathways activating cellular senescence. Int J Biochem Cell Biol. 2005;37(5):961-976.

3. Sherr CJ. Divorcing ARF and p53: an unsettled case. Nat Rev Cancer. 2006;6(9):663-673.

4. Muñoz-Espín D, et al. Programmed cell senescence during mammalian embryonic development. Cell. 2013;155(5):1104-1118.

5. Storer M, et al. Senescence is a developmental mechanism that contributes to embryonic growth and patterning. Cell. 2013;155(5):1119-1130.

6. Demaria M, et al. An essential role for senescent cells in optimal wound healing through secretion of PDGF-AA. Dev Cell. 2014;31(6):722-733.

7. Krizhanovsky V, et al. Senescence of activated stellate cells limits liver fibrosis. Cell. 2008;134(4):657-667.

8. Krishnamurthy J, et al. Ink4a/Arf expression is a biomarker of aging. J Clin Invest. 2004;114(9):1299-1307.

9. Muñoz-Espín D, Serrano M. Cellular senescence: from physiology to pathology. Nat Rev Mol Cell Biol. 2014;15(7):482-496.

10. Dimri GP, et al. A biomarker that identifies senescent human cells in culture and in aging skin in vivo. Proc Natl Acad Sci USA. 1995;92(20):9363-9367.

11. Baker DJ, et al. Clearance of p16Ink4a-positive senescent cells delays ageing-associated disorders. Nature. 2011;479(7372):232-236

12. Sato S, et al. Ablation of the p16(INK4a) tumour suppressor reverses ageing phenotypes of klotho mice. Nat Commun. 2015;6:7035.

13. Baker DJ, et al. Naturally occurring p16(Ink4a)-positive cells shorten healthy life span. Nature. 2016;530(7589):184-189.

14. Furukawa N, Saito M, Hakoshima T, Kohno K. A diphtheria toxin receptor deficient in epidermal growth factor-like biological activity. J Biochem. 2006;140(6):831-841.

15. Saito M, et al. Diphtheria toxin receptor-mediated conditional and targeted cell ablation in transgenic mice. Nat Biotechnol. 2001;19(8):746-750.

16. Churchman ML, Roig I, Jasin M, Keeney S, Sherr CJ. Expression of arf tumor suppressor in spermatogonia facilitates meiotic progression in male germ cells. PLoS Genet. 2011;7(7):e1002157.

17. Verbeken EK, Cauberghs M, Mertens I, Clement J, Lauweryns JM, Van de Woestijne KP. The senile lung. Comparison with normal and emphysematous lungs. 2. Functional aspects. Chest. 1992;101(3):800-809.

18. Verbeken EK, Cauberghs M, Mertens I, Clement J, Lauweryns JM, Van de Woestijne KP. The senile lung. Comparison with normal and emphysematous lungs. 1. Structural aspects. Chest. 1992;101(3):793-799.

19. Huang K, Rabold R, Schofield B, Mitzner W, Tankersley CG. Age-dependent changes of airway and lung parenchyma in C57BL/6J mice. J Appl Physiol. 2007;102(1):200-206.

20. Hirai T, et al. Age-related changes in the static and dynamic mechanical properties of mouse lungs. Respir Physiol. 1995;102(23):195-203

21. Nagase T, Fukuchi Y, Teramoto S, Matsuse T, Orimo H. Mechanical interdependence in relation to age: effects of lung volume on airway resistance in rats. J Appl Physiol. 1994;77(3):1172-1177.

22. Escolar JD, Tejero C, Escolar MA, Montalvo F, Garisa R. Architecture, elastic fiber, and collagen in the distal air portion of the lung of the 18-month-old rat. Anat Rec. 1997;248(1):63-69. 
23. Yamamoto $\mathrm{Y}$, et al. Morphology of aging lung in F344/N rat: alveolar size, connective tissue, and smooth muscle cell markers. Anat Rec A Discov Mol Cell Evol Biol. 2003;272(2):538-547.

24. Knudsen L, Weibel ER, Gundersen HJ, Weinstein FV, Ochs M. Assessment of air space size characteristics by intercept (chord) measurement: an accurate and efficient stereological approach. J Appl Physiol. 2010;108(2):412-421.

25. Kawakami M, Paul JL, Thurlbeck WM. The effect of age on lung structure in male BALB/cNNia inbred mice. Am J Anat. 1984;170(1):1-21.

26. Freund A, Orjalo AV, Desprez PY, Campisi J. Inflammatory networks during cellular senescence: causes and consequences Trends Mol Med. 2010;16(5):238-246.

27. Coppé JP, Desprez PY, Krtolica A, Campisi J. The senescence-associated secretory phenotype: the dark side of tumor suppression. Annu Rev Pathol. 2010;5:99-118.

28. Löffek S, Schilling O, Franzke CW. Series "matrix metalloproteinases in lung health and disease": Biological role of matrix metalloproteinases: a critical balance. Eur Respir J. 2011;38(1):191-208.

29. Murphy G, Cockett MI, Ward RV, Docherty AJ. Matrix metalloproteinase degradation of elastin, type IV collagen and proteoglycan. A quantitative comparison of the activities of $95 \mathrm{kDa}$ and $72 \mathrm{kDa}$ gelatinases, stromelysins -1 and -2 and punctuated metalloproteinase (PUMP). Biochem J. 1991;277 (Pt 1):277-279.

30. Hautamaki RD, Kobayashi DK, Senior RM, Shapiro SD. Requirement for macrophage elastase for cigarette smoke-induced emphysema in mice. Science. 1997;277(5334):2002-2004.

31. Coppé JP, et al. A human-like senescence-associated secretory phenotype is conserved in mouse cells dependent on physiological oxygen. PLoS ONE. 2010;5(2):e9188.

32. Krimpenfort P, Quon KC, Mooi WJ, Loonstra A, Berns A. Loss of p16Ink4a confers susceptibility to metastatic melanoma in mice. Nature. 2001;413(6851):83-86.

33. Sharpless NE, et al. Loss of p16Ink4a with retention of p19Arf predisposes mice to tumorigenesis. Nature. 2001;413(6851):86-91

34. Kamijo T, et al. Tumor suppression at the mouse INK4a locus mediated by the alternative reading frame product p19ARF. Cell. 1997;91(5):649-659.

35. Dimri GP, Itahana K, Acosta M, Campisi J. Regulation of a senescence checkpoint response by the E2F1 transcription factor and p14(ARF) tumor suppressor. Mol Cell Biol. 2000;20(1):273-285.

36. del Arroyo AG, et al. E2F-dependent induction of p14ARF during cell cycle re-entry in human T cells. Cell Cycle. 2007;6(21):2697-2705.

37. Acosta JC, et al. A complex secretory program orchestrated by the inflammasome controls paracrine senescence. Nat Cell Biol. 2013;15(8):978-990.

38. Rodier F, et al. Persistent DNA damage signalling triggers senescence-associated inflammatory cytokine secretion. Nat Cell Biol. 2009;11(8):973-979.

39. Gibbs J, et al. An epithelial circadian clock controls pulmonary inflammation and glucocorticoid action. Nat Med 2014;20(8):919-926.

40. Haspel JA, et al. Circadian rhythm reprogramming during lung inflammation. Nat Commun. 2014;5:4753.

41. John-Schuster G, Gunter S, Hager K, Conlon TM, Eickelberg O, Yildirim AO. Inflammaging increases susceptibility to cigarette smoke-induced COPD. [Published online ahead of print May 29, 2015]. Oncotarget. doi: 10.18632/oncotarget.4027

42. Matulionis DH. Chronic cigarette smoke inhalation and aging in mice: 1. Morphologic and functional lung abnormalities. Exp Lung Res. 1984;7(3-4):237-256.

43. Snider GL. Chronic obstructive pulmonary disease: risk factors, pathophysiology and pathogenesis. Annu Rev Med. 1989;40:411-429.

44. Tsuji T, Aoshiba K, Nagai A. Cigarette smoke induces senescence in alveolar epithelial cells. Am J Respir Cell Mol Biol. 2004;31(6):643-649.

45. Nyunoya T, Monick MM, Klingelhutz A, Yarovinsky TO, Cagley JR, Hunninghake GW. Cigarette smoke induces cellular senescence. Am J Respir Cell Mol Biol. 2006;35(6):681-688.

46. van Deursen JM. The role of senescent cells in ageing. Nature. 2014;509(7501):439-446.

47. Childs BG, Durik M, Baker DJ, van Deursen JM. Cellular senescence in aging and age-related disease: from mechanisms to therapy. Nat Med. 2015;21(12):1424-1435.

48. Kirkland JL, Tchkonia T. Clinical strategies and animal models for developing senolytic agents. Exp Gerontol. 2015;68:19-25.

49. Zhu Y, et al. The Achilles' heel of senescent cells: from transcriptome to senolytic drugs. Aging Cell. 2015;14(4):644-658

50. Chang J, et al. Clearance of senescent cells by ABT263 rejuvenates aged hematopoietic stem cells in mice. Nat Med. 2016;22(1):78-83

51. Muyrers JP, Zhang Y, Benes V, Testa G, Ansorge W, Stewart AF. Point mutation of bacterial artificial chromosomes by ET recombination. EMBO Rep. 2000;1(3):239-243.

52. Abe K, Hazama M, Katoh H, Yamamura K, Suzuki M. Establishment of an efficient BAC transgenesis protocol and its application to functional characterization of the mouse Brachyury locus. Exp Anim. 2004;53(4):311-320.

53. Zindy F, et al. Myc signaling via the ARF tumor suppressor regulates p53-dependent apoptosis and immortalization. Genes Dev. 1998;12(15):2424-2433

54. Katwa P, et al. A carbon nanotube toxicity paradigm driven by mast cells and the IL-(3)(3)/ST(2) axis. Small. 2012;8(18):2904-2912.

55. Shalaby KH, Gold LG, Schuessler TF, Martin JG, Robichaud A. Combined forced oscillation and forced expiration measurements in mice for the assessment of airway hyperresponsiveness. Respir Res. 2010;11:82.

56. Kawagishi $\mathrm{H}$, et al. HuR maintains a replicative life span by repressing the ARF tumor suppressor. Mol Cell Biol. 2013;33(10):1886-1900

57. McQualter JL, Yuen K, Williams B, Bertoncello I. Evidence of an epithelial stem/progenitor cell hierarchy in the adult mouse lung. Proc Natl Acad Sci USA. 2010;107(4):1414-1419.

58. Kanehisa M, Goto S. KEGG: kyoto encyclopedia of genes and genomes. Nucleic Acids Res. 2000;28(1):27-30.

59. Kanehisa M, Goto S, Sato Y, Kawashima M, Furumichi M, Tanabe M. Data, information, knowledge and principle: back to metabolism in KEGG. Nucleic Acids Res. 2014;42(Database issue):D199-D205. 